\title{
Interaction potential and near wall dynamics of spherical colloids in suspensions of rod-like fd-virus.
}

\author{
Peter Holmqvist, Dzina Kleshchanok and Peter R. Lang ${ }^{\mathrm{a}}$ \\ Forschungszentrum Jülich, Institut für Festkörperforschung, 52425 Jülich, Germany \\ Received: date / Revised version: date
}

\begin{abstract}
Averaged diffusivities of spherical colloids in solutions of rod-like particles, i. e. fd-virus, were measured with EWDLS and TIRM. While the experimentally observed near wall dynamics of the spheres in the absence of fd are well described by standard hydrodynamic theories, there are significant deviations at finite $\mathrm{fd}-$-concentrations. Both experimental methods yield data which are significantly smaller than the theoretical predictions.
\end{abstract}

PACS. 82.70.Dd colloids $-78.35 .+\mathrm{c}$ Brillouin and Rayleigh scattering; other light scattering $-68.08 .-\mathrm{p}$ Liquid-solid interfaces

\section{Introduction}

To a solution of colloidal particles a hard wall presents a severe perturbation of the static and the hydrodynamic interaction field. Theoretically it is long known $[1,2]$ that the diffusivity of a spherical particle in the ultimate vicinity of a wall will tend to zero due to the so called wall drag effect, if stick boundary conditions apply. This theoretical decription was cast into its nowadays widely accepted form by Brenner et al. [3,4]. On the other hand, the Derjaguin approximation [5] predicts that any kind of static interaction potential between a wall and a spherical particle should have a contact potential which is twice as large as the same kind of potential between two equally sized spheres of the same material. Although well accepted, the direct experimental verification of these predictions became possible only during the last two decades. Based on the pioneering work of Lan et al. [6] dynamic light scattering with evanescent wave (EWDLS) illumination was developed to a powerful tool for the investigation of near wall colloidal dynamics. EWDLS has been used several times to study the dynamics of colloidal spheres close to a glass-solution interface. Garnier and Ostrowsky [7] measured a reduction of the weighted average particle diffusion coefficient close to a wall, which was supported by Brownian dynamics simulations. Hosoda and coworkers [8] showed that the particle mobility close to a wall is anisotropic for polystyrene colloids. With a novel kind of experimental setup, which allows to vary the magnitude of the scattering vector parallel, $Q_{\|}$, and normal, $Q_{\perp}$, to the interface independently, Holmqvist et al. $[9,10]$ found the diffusivities of polystyrene latex particles with radii as

Send offprint requests to: $\mathrm{P}$. Lang

a e-mail: p.lang@fz-juelich.de small as $27 \mathrm{~nm}$ in both directions to be in agreement with the theoretical predictions. Brenner's predictions were also verified by a number of investigations where modern microscopy techniques have been applied. Lin et al. showed that density matched PMMA particles behave as theoretically expected, if the electrostatic interactions are sufficiently screened [11]. Using fluorescence correlation spectroscopy Joly et al. investigated the influence of surface wettability on the dynamics of $100 \mathrm{~nm}$ particles confined between two flat interfaces [12]. These authors found excellent agreement of the experimental data with hydrodynamic predictions for wettable surfaces, while deviations occur for partially wettable surfaces, i. e. when the stick boundary conditions are violated. If static interaction of the particles with the wall are non-negligible, further deviations from the expected behavior of hard spheres will occur. Khim et al. [13,14] reported for charge stabilized colloids that the mobility normal to the wall was much smaller than expected. According to the authors this is due to particle wall interactions other than excluded volume and hydrodynamics. As an experimental determination of the particle wall interaction is possible with total internal reflection microscopy (TIRM) [15], its effect can in principle be accounted for in the analysis of experiments investigating colloidal near wall diffusivity [10].

In this contribution we report on experimental investigation of the depletion interaction potential between polystyrene latex particles and an adjacent glass wall mediated by rodlike fd-virus particles and the effect of this potential on the sphere dynamics near the wall. We find that the diffusivity of the spheres, both normal and parallel to the wall, is reduced by the presence of the fd-virus, as one would intuitively expect if there is an attractive potential between the spheres and the wall. However, the effect is much stronger than model calculations based on the static 
potential predict. We therefore conclude that we observed an effect of the rod-like co-solute on the colloids' dynamics, which can not be explained by the effect of a static depletion potential. We conjecture that Brenner's prediction, which was originally devised to describe the hydrodynamic interaction of a single sphere and a wall, will fail if a second species of colloidal size is present in the solution and that the rods have to be regarded as a second hydrodynamically active component on equal footing with the spherical particles.

\section{Experimental}

\subsection{Measurement principles}

The technical details of the experimental setups we used for the reported investigation have been described in great detail elsewhere $[10,16,17]$. Therefore, we will give only a brief introduction to the measurement principles here.

\subsubsection{Total Internal Reflection Microscopy (TIRM)}

The interaction potentials between a single colloidal particle and a wall can be obtained by analysing the fluctuations of the light which is scattered from an evanescent field by a single particle [15]. The technique utilizes Boltzmann's law, which connects the probability density of finding the particle at a certain separation distance, $h$, with its potential energy at this distance

$$
p(h)=A \exp \left\{-\beta \phi_{t o t}(h)\right\}
$$

where $A$ is a normalizing constant, $\beta$ the inverse thermal energy and $\phi_{\text {tot }}(h)$ the potential between the particle and the interface from which the laser beam is reflected. If the potential has a shallow minimum at a separation distance, $h_{\min }$, the particle will sample a distribution of distances around $h_{m i n}$ due to Brownian motion. These fluctuations can be directly observed in a TIRM-experiment. For this purpose a laser beam is directed via a prism onto the glass/solution interface with an incident angle, $\alpha_{i}$, greater than the critical angle of total reflection. The electric field of the laser beam penetrates the interface causing an evanescent wave, the amplitude of which decays exponentially along the interface normal, and a single colloidal sphere, interacting with this evanescent wave, will scatter the light with strength depending on its position as [18, $19]$

$$
I(h)=I_{0} \exp \{-\Lambda h\}
$$

where $\Lambda^{-1}=4 \pi / \lambda \sqrt{n_{1}^{2} \sin ^{2}\left(\alpha_{i}\right)-n_{2}^{2}}$ is the inverse penetration depth of the evanescent intensity, $\lambda$ is the laser wave length and $n_{1}$ and $n_{2}$ are the refrective indices of the glass and the solvent, respectively. A photomultiplier is used to monitor the scattered intensity as a function of time. For a sufficiently high number of data points (typically more than $\left.5 \times 10^{4}\right)$ the histogram $N([I(h)]$ of intensities converges to the probability density distribution of the intensity $p[I(h)]$. The latter is directly related to the probability density of separation distances by

$$
p(h)=p[I(h)] \frac{d I}{d h}=-\frac{1}{\Lambda} p[I(h)] I(h) \approx-\frac{1}{\Lambda} N[I(h)] I(h)
$$

This can be converted into a potential energy profile using Boltzmann's law which gives

$$
\beta \Delta \phi(h)=\ln \frac{N\left[I\left(h_{\min }\right)\right] I\left(h_{\min }\right)}{N[I(h)] I(h)}
$$

where $\Delta \phi(h)$ is the potential difference with respect to the minimum. To determine absolute separation distances the scattering intensity for the particle at zero separation distance $I(h=0)$ has to be known, because

$$
h=\frac{1}{\Lambda} \ln \frac{I(h=0)}{I(h)} .
$$

Commonly this is obtained by screening the Coulombic repulsion between the sphere and the wall and thereby bringing the sphere into contact with the wall.

A different route to determine the separation distance was proposed by Frej et al. [20] and improved by Bevan et al. [21]. This method, sometimes referred to as hydrodynamic separation method, is based on the information on the particle dynamics which is contained in the time dependence of the scattered intensity. From the initial slope of the normalized time auto-correlation function of the scattered intensity an averaged apparent diffusion coefficient of the particle normal to the wall can be obtained as

$D_{\perp}^{T I R M}=-\Lambda^{-2} \frac{\partial g_{2}(t \rightarrow 0) / \partial t}{g_{2}(0)}=\frac{\int_{-\infty}^{\infty} d h D_{0} f_{\perp}(z) I(h)^{2} p(h)}{\int_{-\infty}^{\infty} d h I(h)^{2} p(h)}$.

Here $D_{0} f_{\perp}(z)$ is the position depending diffusivity of the particle normal to the wall as predicted by Brenner [3], with $D_{0}$ the bulk diffusion coefficient and $f_{\perp}(z)$ accounting for the wall drag effect. The particle center position $z=h+R$ with the particle radius $R$. For experimental purposes the integral is approximated by

$$
g_{2}(j \Delta \tau)=\frac{1}{n-j} \sum_{i=1}^{n-j} I(i \Delta \tau) I((i+j) \Delta \tau)
$$

where $\Delta \tau$ is the lapse of time between two sequential observations, $n$ is their total number, $i$ refers to a specific observation and $j$ to the delay time. In their very comprehensive article Bevan and Prieve [22] describe how to determine $h_{\min }$, if $D_{\perp}^{T I R M}$ is known. In this contribution we will not use this approach, our aim is rather to compare the dynamic data available from TIRM-experiments to those data which were measured independently with EWDLS.

\subsubsection{Evanescent Wave Dynamic Light Scattering (EWDLS)}

We have recently improved the experimental and theoretical basis of evanescent wave dynamic light scattering (EWDLS) $[9,10]$, in a way that allows us to study 
the anisotropy of the near wall dynamics of particles as small as ca. $25 \mathrm{~nm}$ in radius. In EWDLS-experiments the evanescent wave, which is created upon total reflection of the laser beam is used as the incident radiation for a dynamic light scattering experiment. Different from TIRMexperiments, here the light scattered by an ensemble of particles is detected and analysed. With our instrumental design it is possible to vary the amount of the normal, $Q_{\perp}$, and the parallel, $Q_{\|}$, component, of the scattering vector (that is the difference between the wave vector of the scattered wave and the evanescent wave, $\mathbf{Q}=\mathbf{k}_{S}-\mathbf{k}_{e}$ ) independently. By this means it becomes possible to distinguish between the particle mobility parallel and normal to the interface. We showed [9] that the initial decay rate, $\Gamma$, of the time auto correlation function of the scattered field, $g_{1}(t)$, depends on the scattering vector components as

$$
\Gamma=\left\langle D_{\|}\right\rangle Q_{\|}^{2}+\left\langle D_{\perp}\right\rangle\left(Q_{\perp}^{2}+4 / \Lambda^{2}\right),
$$

if hydrodynamic and static interaction between the colloidal particles and the wall are taken into account via the definition of the mean diffusivity components.

$$
\begin{aligned}
& \frac{\left\langle D_{\|, \perp}\right\rangle}{D_{0}}= \\
& \frac{\int_{z>R} d z \exp \{-\beta \phi(z)\} \exp \{-(z-R) \Lambda / 2\} f_{\|, \perp}(z)}{\int_{z>R} d z \exp \{-\beta \phi(z)\} \exp \{-(z-R) \Lambda / 2\}} .
\end{aligned}
$$

These integrations are performed inherently in the experiments, due to the nature of the evanescent illumination. Therefore $\left\langle D_{\|, \perp}\right\rangle$ can be measured by linear least squares fitting of $\Gamma$ vs $Q_{\|}$at fixed $Q_{\perp}$ and vice versa. Note that $\left\langle D_{\perp}\right\rangle$ and $D_{\perp}^{T I R M}$ are differently weighted means of the same quantity.

\subsection{Sample Preparation}

TIRM-experiments were performed with polystyrene sulfonate (PS) latex probe particles with a radius of $R=$ $1.5 \mu \mathrm{m}$ and a standard deviations of $\sigma<0.1$, which were purchased from Polyscience Inc., U.S.A. The particles were diluted with TRIS buffer of $\mathrm{pH}=8.2$ from the stock suspension down to a volume fraction of ca. $10^{-9}$ for the experiments. The solutions were contained in a carbonized PTFE frame sandwiched between two microscope slides from BK-7 glass, which were received from Fischer Scientific Co., U.S.A. The glass slides were thoroughly cleaned in an ultrasonic bath for $30 \mathrm{~min}$ in ethanol before assembling the sample cell. The buffer concentration was adjusted to $1.2 \mathrm{mMol} / \mathrm{L}$ (note that at $\mathrm{pH}=8.2$ the concentration of the dissociated ions is $0.6 \mathrm{mMol} / \mathrm{L}$ ) corresponding to a nominal Debye screening length of $\kappa^{-1}=12.4$ $\mathrm{nm}$. This value was chosen for convenience, in order to adjust the position of the potential minimum in the range of $h_{\min } \approx 100-150 \mathrm{~nm}$.

The EWDLS-experiments were carried out on aqueous buffer solutions, containing $20 \mathrm{mMol} / \mathrm{L}$ TRIS (corresponding to an ion concentration of $10 \mathrm{mMol} / \mathrm{L}$ ), of polystyrene latex spheres (Interfacial Dynamics Corp., Portland, USA) with a radius of $\mathrm{R}=85 \mathrm{~nm}$. The beads were diluted from their stock solutions to a volume fraction of $2 \times 10^{-4}$. At these conditions the particles may be regarded as hard spheres, since the Debye screening length is in the range of $3 \mathrm{~nm}$, while the mean interparticle distance is of the order of several thousand nanometers.

In both, TIRM- and EWDLS-experiments bacteriophage fd-virus were used to introduce an attractive depletion potential between the spherical colloids and the wall. fd-virus are rod-like particles with a length of $L=$ $880 \mathrm{~nm}$, a cross section diameter of $d_{c s}=6.6 \mathrm{~nm}$ and a molar mass of $M_{r}=1.64 \times 10^{7} \mathrm{~g} / \mathrm{Mol}$. From geometrical considerations this yields an overlap concentration $c^{*} \approx 7.5 \times 10^{-2} \mathrm{~g} / \mathrm{L}$. The virus particles were produced in our laboratory following standard procedures [23].

\section{Results and discussion}

The analysis of the EWDLS-data from a suspension of spherical PS particles at zero $\mathrm{fd}$-concentration yields the mean diffusivities, which are displayed in Fig. 1 as open symbols. We present the data as diffusivities normalized to the bulk diffusion coefficient $D_{0}$ vs. the penetration depth normalized by the particle radius. We used $D_{0} \approx 2500$ $\mathrm{nm}^{2} / \mathrm{ms}$ in the present case which is the diffusion coefficient in in absence of the fd corrected with a viscosity increase of about $10 \%$ due to the presence of the virus [24, $25]$. The quantity $2 / \Lambda R$ can be interpreted as a measure for the average particle to wall separation distance. Within experimental error the results follow nicely the theoretical curves, which were calculated by numerical integration according to eq. 9 using

$$
\begin{aligned}
f_{\perp}(z)^{-1} & =\frac{4 \sinh \alpha}{3} \sum_{n=1}^{\infty} \frac{n(n+1)}{(2 n-1)(2 n+3)} \\
& \times\left[\frac{2 \sinh [(2 n+1) \alpha]+(2 n+1) \sinh [2 \alpha]}{(2 \sinh [(n+1 / 2) \alpha])^{2}-((2 n+1) \sinh \alpha)^{2}}-1\right]
\end{aligned}
$$

and

$$
f_{\|}(z)=\left[1-\frac{9}{16} \frac{R}{z}+\frac{1}{8}\left(\frac{R}{z}\right)^{3}-\frac{45}{256}\left(\frac{R}{z}\right)^{4}-\frac{1}{16}\left(\frac{R}{z}\right)^{5}\right] .
$$

Further we assumed that the wall and the colloids interact solely by electrostatic repulsion when no $\mathrm{fd}$ is present.

If the rod-like virus particles are added to the solution, we have to account for an attractive depletion potential. In the present case, where $L \approx 10 R$ there is no closed analytical form of the depletion potential available. However, as we have shown recently, the latter can be fairly well approximated by

$$
\beta \phi_{\text {depl }}(h) \approx \nu\left(1-\frac{h}{L}\right)^{3}
$$

where $\nu$ is a parameter depending on the rod concentration [26]. In the case of $R \gtrsim 2 L$ when the Derjaguin 


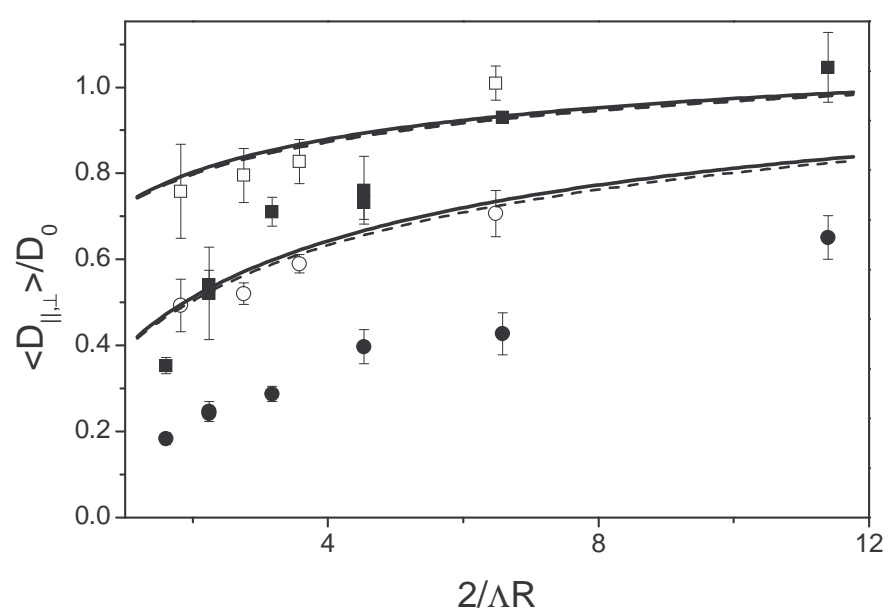

Fig. 1. Mean diffusivities as determined by EWDLS vs normalized penetration depth of the evanescent field. Lines are numerical data according to eq. 9 for zero fd-concentration (full curves) and $c_{f d}=0.17 \mathrm{~g} / \mathrm{L}$ (dashed curves) The top pair of curves corresponds to the diffusivity parallel to the wall while the bottom pair represents the normal component of diffusivity. Symbols are experimental data for the diffusivity parallel (squares) and perpendicular (bullets) to the wall, open symbols refer to zero fd-concentration and full symbols to $c_{f d}=0.17$ $\mathrm{g} / \mathrm{L}$.

approximation is applicable $\nu$ take the form derived by Auvray [27] and Mao et al [28] $\nu=-\pi c_{\text {rod }} N_{A} R L^{2} / 3 M_{r}$ where $c_{\text {rod }}$ is the mass concentration of the rods, $N_{A}$ Avogadro's number and $M_{r}$ the molar mass of the rods. Thus the total potential entering into eq. 9 is

$$
\beta \phi_{t o t}(h)=B \exp \{-\kappa h\}+\nu\left(1-\frac{h}{L}\right)^{3}
$$

in the superposition approximation. Here $B$ is the amplitude of the electrostatic repulsion, depending on the particle's and the wall's surface charge density and the Debye screening length is related to the salt concentration by $\kappa^{-1}=0.304 / c_{s}^{0.5}$. In the present case $\kappa^{-1} \approx 3 \mathrm{~nm}$ and we choose $\beta B=100$, which is a reasonable value for systems like the one investigated. Nevertheless, due to the high screening even a ten times higher value of $B$ would not produce a discernably different curve in Fig. 1.

The dashed line is calculated for a finite concentration of fd-virus, $c_{f d}=0.17 \mathrm{~g} / \mathrm{L} \approx 2 c^{*}$, which corresponds $\nu \approx 8.6 \times 10^{-2}$. It is evident that the resulting depletion potential shouldn't hardly influence the dynamic properties of the spherical colloids. Nevertheless, the experimental values of diffusivities show a different behavior.

The diffusivity parallel to the wall follows the theoretical prediction at the highest penetration depths, while it is significantly smaller at low penetration depth, that is the more sensitive the experiment is to the near wall $\mathrm{dy}-$ namics, the larger the deviations from eq. 9. These deviations are even more pronounced for the diffusivity normal to the wall. Throughout the whole range of penetration depths, the experimental data are roughly by a factor of two smaller than the predictions by eq. 9. The same gen-

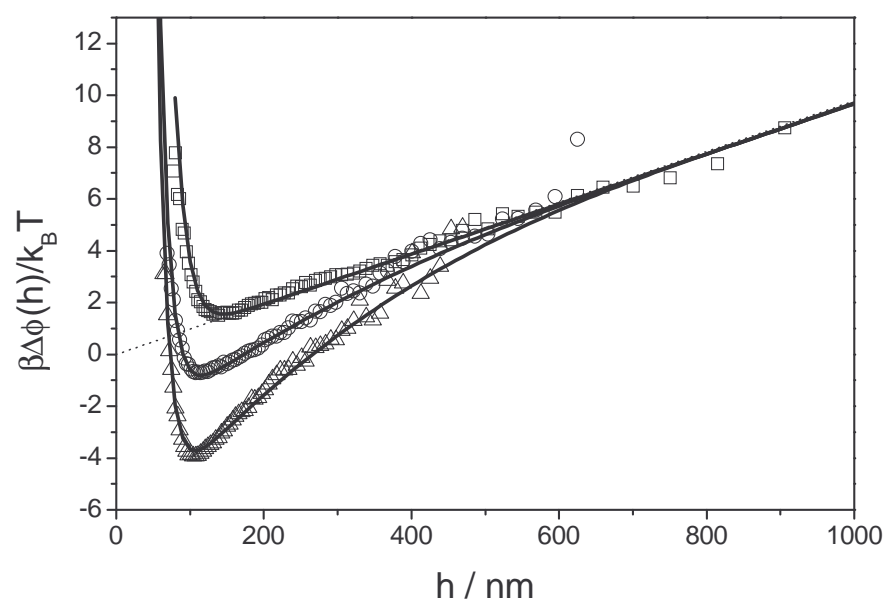

Fig. 2. Sphere wall interaction potentials as determined with TIRM. Symbols are experimental data: $c_{f d}=0$ (squares), $c_{f d}=0.085 \mathrm{~g} / \mathrm{L}$ (circles) and $c_{f d}=0.17 \mathrm{~g} / \mathrm{L}$ (triangles). Full lines are best non-linear least squares fits of eq. 14 to the data. The dotted line represents the gravitational contribution.

eral trend was also observed for an fd-concentration of $c_{f d}=0.05 \mathrm{~g} / \mathrm{L}$. These data are not shown for the sake of clarity. Based on the difference between the experimental data and the theoretical curves, we conclude that the effect of the fd-rods on the spheres' dynamics can not be described, taking into account only the depletion potential which is created by the rods. Rather, it is likely that the rods have to be theoretically treated as a second hydrodynamically active species on equal footing with the spheres.

We realize that a weak point of this argumentation is the lack of an experimental verification of the static interaction potential for the given system. Therefore we performed TIRM-experiments with larger probe spheres of the same chemical nature. In Fig. 2 we show the experimentally determined interaction profiles together with the best non-linear least squares fits of a superposition of three contributions to the total interaction potential, i. e. electrostatic repulsion, gravity and depletion interaction as

$$
\beta \Delta \phi(h)=B \exp \{-\kappa h\}+G_{e f f} h-\frac{\pi c_{f d} N_{A}}{3 M_{r}} R L^{2}\left(1-\frac{h}{L}\right)^{3} .
$$

Here $G_{\text {eff }}$ is the buoyancy corrected gravitational force plus a contribution due to the radiation pressure of an optical trap which was applied to keep the particle laterally in position. The third term is the first order density approximation for the depletion potential mediated by rods in the Derjaguin approximation [27,28]. In order to keep the number of freely floating parameters in the least squares fitting procedure as small as possible, we fixed $R=1500 \mathrm{~nm}, G_{\text {eff }}=41 \mathrm{fN}$ and $L=880$ $\mathrm{nm}$, thereby neglecting the effect of the finite flexibility of the fd-virus [29]. Further we locked the amplitude of the electrostatic repulsion to the value determined by from the experiment in the absence of fd by data fitting, i. e. 


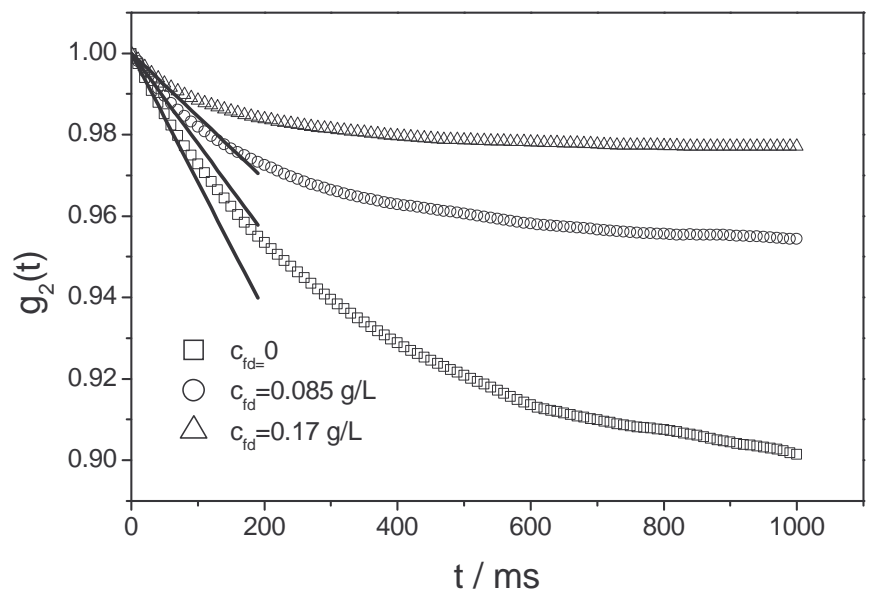

Fig. 3. Time auto-correlation functions of the scattered intensity observed in TIRM-experiments on a PS sphere in the presence $\mathrm{fd}$-virus solutions with different rod concentrations as indicated in the legend.

$\beta B=1380$. Thus, we remained with a single adjustable parameter to fit the potentials in the presence of the rods, which was determined as $\kappa^{-1}=13.1 \pm 0.1 \mathrm{~nm}$.

The intensity traces from which the potentials were calculated, were time auto-correlated according to eq. 7 which resulted in the curves displayed in Fig. 3. The averaged diffusivities normal to the wall $D_{\perp}^{T I R M}$ was determined from the initial slopes of the correlation functions, which are shown as full lines in the plot. With increasing fd concentration $D_{\perp}^{T I R M}$ becomes significantly smaller, which is in line with our EWDLS observations. We shall now compare the experimental data for $D_{\perp}^{T I R M}$ with theoretical predictions. For this purpose we integrated eq. 6 numerically, using the experimentally determined parameters, for the calculation of $\beta \Delta \phi(h)$, i. e. $p(h)$. The resulting averaged diffusivities are plotted as a function of the normalized penetration depth of the evanescent wave in Fig. 4 together with the experimental data. It is obvious that the experimental point and the theoretical curve match rather well for the case where no fd-virus is present in the solution, while deviations occur at finite rod concentrations. To get an estimate for the significance of this deviation, we performed a second set of experiments on a different particle in the absence of $\mathrm{fd}-$-where we varied the penetration depth of the evanescent wave. The resulting $D_{\perp}^{T I R M}$ are shown as open symbols in Fig. 4. From the scatter of the data points obtained in the absence of fd we estimate that the confidence interval of the experimental data is $\pm 10 \%$. This is the size of the error bars in Fig. 5 were we plotted the ratio of the theoretical predictions and the experimental values of $D_{\perp}^{T I R M}$ versus the concentration of the virus.

From this figure it is evident, that the deviation of the experimental values from the theoretical predictions is significant. Further, the dynamic data obtained from TIRM-measurements follow the same trend as the results for the diffusivity normal to the wall, $\left\langle D_{\perp}\right\rangle$, measured in EWDLS-experiments, which are also plotted in

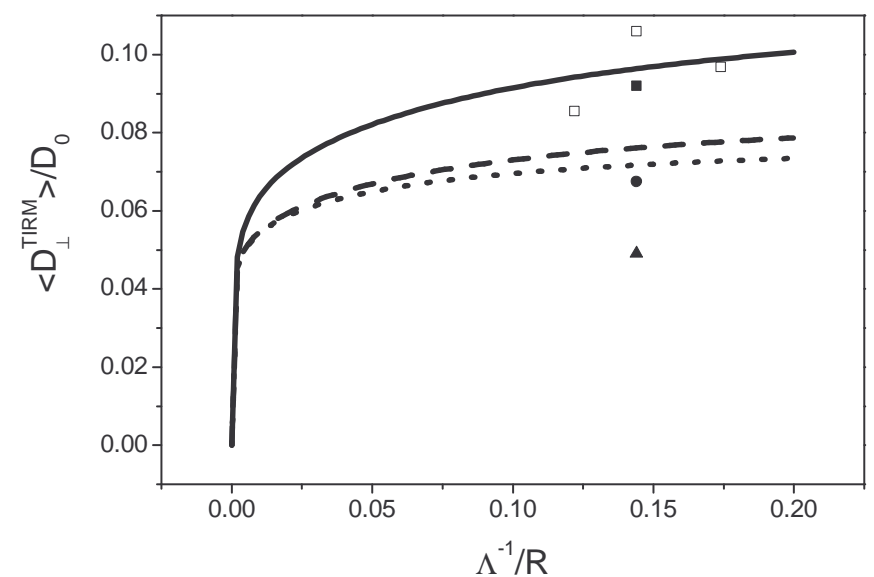

Fig. 4. Averaged diffusivities normal to the wall for a PS sphere in the presence differently concentrated fd-virus solutions. Symbols are experimental data: $c_{f d}=0$ (full squares), $c_{f d}=0.085 \mathrm{~g} / \mathrm{l}$ (bullet) and $c_{f d}=0.17 \mathrm{~g} / \mathrm{L}$ (triangle). Open squares refer to a series of experiments on a different particle in the absence of fd. Lines are numerical calculations according to eq. $6: c_{f d}=0$ (full), $c_{f d}=0.085 \mathrm{~g} / \mathrm{l}$ (dashed) and $c_{f d}=0.17$ $\mathrm{g} / \mathrm{L}($ dotted)

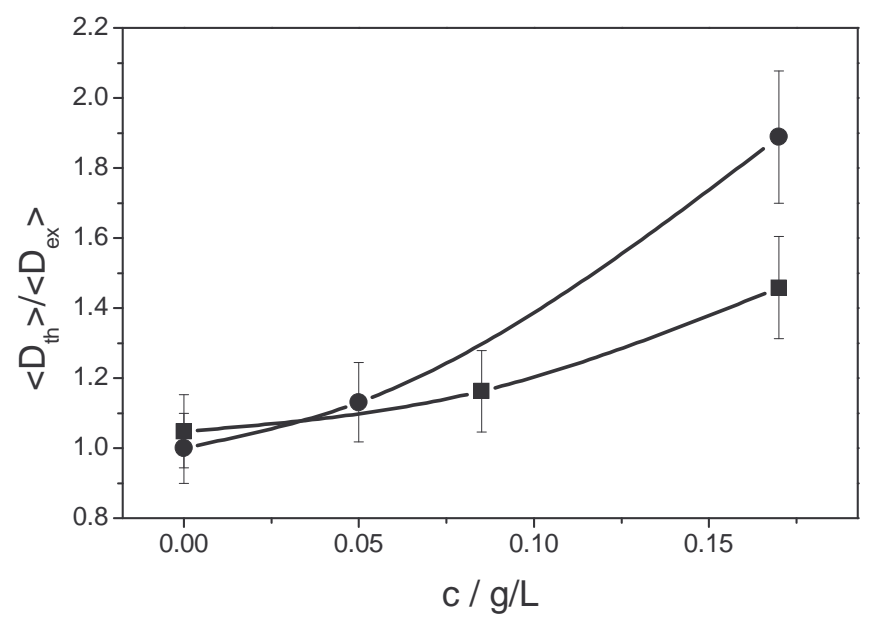

Fig. 5. Ratio of theoretical and experimental averaged diffusivity normal to the wall as a function of $\mathrm{fd}$-concentration as determined by EWDLS (bullets; $1 / \Lambda=240 \mathrm{~nm}$ ) and TIRM (squares; $1 / \Lambda=224 \mathrm{~nm}$ ).

Fig. 5. Note that $D_{\perp}^{T I R M} \neq\left\langle D_{\perp}\right\rangle$, but they are differently weighted means of the same physical property. Thus, the dynamic data obtained by the two methods strongly support each other. This shows positively that it is not possible to describe the influence of the rod-like particles on the near-wall dynamics of colloidal spheres by an effective depletion potential alone. In turn, this implies that Brenner's predictions $[3,4]$, which originally have been derived for the situation of a single sphere approaching a wall in a quiescent fluid, is not suited to describe the near wall dynamics of spherical particles in the presence of a rodlike co-solute. Rather, an additional slowing down of the spheres is observed, which is very likely due to a hydrodynamic effect of the rods. It can be excluded that a wall 
induced structuring of the rod solution is the reason for the observed deceleration of the spheres, because available theoretical [30,31] and experimental [32] data show that wall induced structure formation should only occur at concentrations which are about $90 \%$ of the value needed to drive the isotropic to nematic transition in the bulk. For the case of fd at the buffer concentration used here this would correspond $c_{f d} \approx 12.5 \mathrm{~g} / \mathrm{L}$. It is further important to note, that the additional slowing down is not only observed in situations where the radius of the spherical colloids is much smaller than the rod length, but it also occurs in the opposite limit where $R>L$. Consequently care has to be taken, if dynamic data are used for the determination of absolute distances for potential profiles determined in TIRM-experiments. It has to be checked, that Brenner's predictions reproduce the experimental $D_{\perp}^{T I R M}$ values within experimental scatter. Otherwise the hydrodynamic separation method will inevitably lead to erroneous results.

\section{Conclusions}

EWDLS and TIRM was used to measure averaged diffusivities of spherical colloids in solution containing different concentrations of rod-like fd-virus particles. The experimentally observed near wall dynamics of the spheres are well described by standard hydrodynamic theories, if there are no rods present. On the other hand, pronounced deviations occur at finite $\mathrm{fd}$-concentrations. Both experimental methods yield data which are significantly smaller than the theoretical predictions. This shows that the effect of the rods on the spheres' diffusivity can not be described by an effective depletion potential alone. The rods have rather to be treated as a co-solute on an equal footing with the spheres, which couples hydrodynamically to the mobility of the spheres. Since to our best knowledge there is no theory available for this situation, we are currently running computer simulations in order to gain further insight to this problem.

\section{Acknowledgement}

We thank K. Sellinghoff and P. Lettinga for making the fd-virus samples available.

\section{References}

1. H. A. Lorentz, Abh. Theor. Phys. 1, 23 (1907)

2. H. Faxén, Ark. Mat. Astrom. Fys. 17, 1 (1923)

3. H. Brenner, Chem. Eng. Sci 16, 242 (1961)

4. A. J. Goldman, R. G. Cox, H. Brenner, Chem. Eng. Sci. 22, 637 (1967)

5. B. V. Derjaguin, Kolloid Zeits. 69, 155 (1934)

6. K. H. Lan, N Ostrowsky, D. Sornette, Phys. Rev. Lett. 57, 17 (1986)

7. N. Garnier, N. Ostrowsky, J. Phys. II France 1, 1221 (1991)
8. M. Hosoda, K. Sakai, K. Takagi, Phys. Rev. E 58, 6275 (1998)

9. P. Holmqvist, J. K. G. Dhont, P. R. Lang, Phys. Rev. E 74, $021402(2006)$

10. P. Holmqvist, J. K. G. Dhont, P. R. Lang, J. Chem. Phys. 126, 044707 (2007)

11. B. Lin, J. Yu, S. A. Rice, Phys. Rev. E 62, 3909 (2000)

12. L. Joly, C. Ybert, L. Bocquet, Phys. Rev. Lett. 96, 046101 (2006)

13. K. D. Kihm, A. Banerjee, C.K. Choi, T. Tagaki, Experiments in Fluids 37, 811 (2004)

14. A. Banerjee, K. D. Kihm, Phys. Rev. E 72, 042101 (2005)

15. D. C. Prieve, Advances in Colloid and Interface Science 82, 93 (1999)

16. D. Kleshchanok, J. E. Wong, R. von Klitzing, P. R. Lang, Progr. Colloid Polym. Sci 133, 52 (2006)

17. D. Kleshchanok, R. Tuinier, P. R. Lang, Langmuir 22, 9121 (2006)

18. D. C. Prieve, J. Y. Walz, Applied Optics 32, 1629 (1993)

19. H. Chew, D. S. Wang, M. Kerker, Appl.Opt. 18, 2679 (1979)

20. N. A. Frej, C. D. Prieve, J. Chem. Phys. 98, 7552 (1993)

21. M. A. Bevan, C. D. Prieve, J. Chem. Phys. 113, 1228 (2000)

22. M. A. Bevan, D. C. Prieve, Langmuir 15, 7925 (1999)

23. T. Maniantis, J. Sambrook, E. F. E. Fritsch, Molecular Cloning (Cold Spring Harbor University Press, 1989)

24. C. Graf, H. Kramer, M. Deggelmann, M. Hagenbchle, C. Johner, C. Martin, R. Weber, J. Chem. Phys. 98, 4920 (1993)

25. K. Kang, J. Gapinski, M. P. Lettinga, J. Buitenhuis, G. Meier, M. Ratajczyk, J. K. G. Dhont, A. Patkowski, J. Chem. Phys. 122, 044905 (2005)

26. P. R. Lang, J. Chem. Phys. 127, 124906 (2007)

27. L. Auvray, J. Phys. 42, 79 (1981)

28. Y Mao, M.E. Cates, H. N. W. Lekkerkerker, J. Chem. Phys. 106, 3721 (1997)

29. A. W. C. Lau, K.-H. Lin, Yodh A. G. , Phys. Rev. E 66, $020401(2002)$

30. R. van Roij, M. Dijkstra, R. Evans, J, Chem. Phys. 113, 7689 (2000)

31. M. Dijkstra, R. van Roij, R. Evans, Phys. Rev. E 63, 51703 (2001)

32. P. Lang, L. Willner, W. Pyckhout-Hintzen, R. Krastev, Langmuir 19, 7597 (2003) 